\title{
Some Extensions of Legendre Quadrature
}

\author{
By A. C. R. Newbery
}

Abstract. The $m$-point Gauss-Legendre formula gives an exact expression for the integral of an algebraic polynomial of maximum degree $2 m-1$ in terms of $m$ ordinates. It is shown that analogous formulas can be derived for exponential and trigonometric polynomials.

The Gauss-Legendre quadrature formulas approximate the integral of a function by a weighted sum of function-values. When $m$ function-values are used, the formula is exact for functions belonging to a specific $2 m$-dimensional space, namely the polynomials of degree zero through $2 m-1$. This finite-dimensional space is in no sense 'representative' of the infinite space over which the formula gives exact results. The latter space includes all Riemann-integrable functions which are odd with reference to an origin located at the mid-point of the domain of integration; it also includes an infinite class of even functions, and this class contains an infinite subclass of polynomials. Similar remarks also apply to the Newton-Cotes formulas. In [1] there is discussion of weighted quadrature formulas of the form

$$
\int_{a}^{b} f(x) d x=\sum_{j=1}^{m} \beta_{j} g\left(x_{j}\right)+T, \text { where } f(x)=g(x) w(x),
$$

$w(x)$ is a fixed nonnegative weight function, the $\beta_{j}$ are the Christoffel numbers associated with $w$, and the truncation error $T$ vanishes when $g(x)$ is a polynomial of degree not exceeding $2 m-1$. It is shown there that

$$
\beta_{j}=\frac{1}{q_{m}^{\prime}\left(x_{j}\right)} \int_{a}^{b} \frac{q_{m}(x)}{\left(x-x_{j}\right)} w(x) d x,
$$

where $q_{m}$ is the $m$ th degree polynomial belonging to a set which is orthogonal with respect to the inner product $\left(q_{r}(x), q_{s}(x)\right) \equiv \int_{a}^{b} q_{r}(x) q_{s}(x) w(x) d x$, and the $x_{j}$ are zeros of $q_{m}$.

Using these results as a basis, we now consider the problem of constructing an $m$-point quadrature formula which gives exact results whenever the integrand $f(x)$ belongs to a $2 m$-dimensional space distinct from those considered in [1]. A simple example is provided by the case where $f(x)$ is a linear combination of exponentials $e^{r x}$, where $r$ takes consecutive (possibly negative) integer values. In this case by writing $e^{x}=z$ we obtain $\int_{a}^{b} f(x) d x=\int_{e^{a}}^{e^{b}} z^{s} g(z) d z$, where $s$ is an integer and $g$ a polynomial. The latter integral is in the form (1), so one can find an $m$-point formula which is valid for up to $2 m$ terms in the linear combination. Specifically, if $r$ takes integer values from $p$ through $p+2 m-1$, then $s=p-1$; the polynomial $q_{m}$ and numbers $\beta_{j}$ are defined as in (1), (2) with $w(z)=z^{s}$ and integration limits $e^{a}, e^{b}$. It then follows that

Received February 16, 1968. 


$$
\int_{a}^{b} f(x) d x=\sum_{1}^{m} \beta_{j} g\left(z_{j}\right)=\sum_{1}^{m} \beta_{j} z_{j}^{-1-s} f\left(\ln z_{j}\right)
$$

equivalently:

$$
\int_{a}^{b} f(x) d x=\sum_{1}^{m} \beta_{j}{ }^{\prime} f\left(x_{j}\right)+T, \quad \text { where } \beta_{j}{ }^{\prime}=\beta_{j} z_{j}^{-1-s},
$$

$x_{j}=\ln z_{j}$, and $T$ is zero whenever $f(x)$ belongs to the specified space.

Another simple case arises when $f(x)$ is a sine polynomial to be integrated from zero to $\pi$. On making the substitution $\cos \theta=x$, and noting that for integers $r$, $\sin r \theta / \sin \theta$ is then an algebraic polynomial in $x$, we obtain

$$
\int_{0}^{\pi} f(\theta) d \theta=\int_{-1}^{1}(f(\theta) / \sin \theta) d x=\int_{-1}^{1} p(x) d x,
$$

where $p(x)$ is an algebraic polynomial. This leads to the formula

$$
\int_{0}^{\pi} f(\theta) d \theta=\int_{1}^{m} \beta_{j}^{\prime} f\left(\theta_{j}\right)+T,
$$

where $T$ is zero whenever $f(\theta)$ is a sine polynomial of degree not exceeding $2 m$. The numbers $\beta_{j}{ }^{\prime}$ and nodes $\theta_{j}$ are related to the standard Legendre numbers $\beta_{j}$ and nodes $x_{j}$ by $\cos \theta_{j}=x_{j}$ and $\beta_{j}{ }^{\prime}=\beta_{j} / \sin \theta_{j}$. It would clearly be inappropriate to use this formula on an integrand that is not well approximated by a sine polynomial. In particular, the formula gives inexact results when $f(\theta)$ is a nonzero constant.

A more difficult problem arises in the case where $f(\theta)$ is a full Fourier series, and the domain of integration is [- $-\alpha \pi, \alpha \pi],|\alpha|<1$. In the case $\alpha=1$ it is known [2] that the parabolic rule is optimal. Let $f(\theta)=\sum_{1}^{n} a_{r} \sin r \theta+\sum_{0}^{n} b_{r} \cos r \theta$. We seek Christoffel numbers $\beta_{j}{ }^{\prime}$ and nodes $\theta_{j}$ such that $\int_{-\alpha \pi}^{\alpha \pi} f(\theta) d \theta=\sum_{1}^{m} \beta_{j}{ }^{\prime} f\left(\theta_{j}\right)$, where $m$ is as small as possible and the $a_{r}, b_{r}$ are arbitrary. If we make the substitutions

$$
x=\tan \theta / 2, \quad \sin \theta=2 x /\left(1+x^{2}\right), \quad \cos \theta=\left(1-x^{2}\right) /\left(1+x^{2}\right),
$$

then the integrand becomes a rational function of $x$, and we have

$$
\int_{-\alpha \pi}^{\alpha \pi} f(\theta) d \theta=\int_{-u}^{u} \frac{P_{2 n}(x)}{\left(1+x^{2}\right)^{n+1}} d x
$$

where $P_{2 n}(x)$ is an algebraic polynomial of degree $2 n$, and $u=\tan \alpha \pi / 2$. Since an $m$-point formula will handle weighted polynomials of degree up to $2 m-1$, we shall need $n+1$ nodes in order to guarantee exact results when the integrand is of the type (6). Now that the problem has been reformulated into one of the type (1) we know that it is possible to derive an appropriate formula by applying (2). This method of derivation has the merit that we know in advance that formulas of all orders do exist; on the other hand a method of undetermined coefficients may be preferable for the purpose of actually constructing the formulas. In applying the latter method, we assume that all nodes and Christoffel numbers are symmetric about the origin, so that there is no need to take further account of the sine component of $f(\theta)$. We will describe the construction of a $2 p$-point formula; this means that we are seeking $2 p$ nodes $\pm \theta_{i}$ and $p$ Christoffel numbers $\beta_{i}$, such that for all integers $r \in[0,2 p-1]$ 


$$
\int_{-\psi}^{\psi} \cos r \theta d \theta=2 \sum_{i=1}^{p} \beta_{i} \cos r \theta_{i}, \quad \text { where }|\psi|=|\alpha \pi|<\pi .
$$

If we define $u_{i}=\cos \theta_{i}, q_{r}=(1 / 2) \int_{-\psi}{ }^{\psi} \cos r \theta d \theta$, then the above equations take the form

$$
\sum_{1}^{p} \beta_{i} T_{r}\left(u_{i}\right)=q_{r}, \quad r=0, \cdots, 2 p-1,
$$

where $T_{r}(u)$ is the $r$ th degree Chebyshev polynomial. Now for $r \geqq 2$ we pick up the Eq. (7) involving $T_{r}$ and replace it by the average of itself and the equation involving $T_{r-2}$. Using the fact that $T_{r}(u)+T_{r-2}(u)=2 u T_{r-1}(u)$ we have therefore "traded" an equation involving $T_{r}$ for one involving $u T_{r-1}$. This in turn can subsequently be "traded" for one involving $u^{2} T_{r-2}$ etc. After a sufficient number of averagings, the equation system ( 7 ) can be brought into the form which one encounters when using Prony's method for exponential interpolation [3, p. 378]; thus a typical left-hand member of the reformulation of system (7) has the form $\sum_{i=1}^{p} \beta_{i} u_{i}{ }^{r}$. To solve this system we proceed as in [3], first constructing and factoring the polynomial whose roots are $u_{i}$, then solving a system of linear equations to determine the $\beta_{i}$. A minor variation in this procedure makes it applicable to the case where the number of nodes is odd. Formulas for $m=2,3,4$ are as follows, with $\mathrm{s}$, c denoting $\sin \psi, \cos \psi$ :

$$
\begin{aligned}
& \text { For } m=2, u_{1}=\mathrm{s} / \psi, \beta_{1}=\psi \text {. } \\
& \text { For } m=3, u_{0}=1, u_{1}=(1 / 2)(\mathrm{sc}+\psi-2 \mathrm{~s}) /(\mathrm{s}-\psi), \\
& \beta_{0}=2\left(2 \mathrm{~s}^{2}-\psi^{2}-\mathrm{sc} \psi\right) /(4 \mathrm{~s}-3 \psi-\mathrm{sc}), \beta_{1}=2\left(2 \mathrm{~s} \psi-\psi^{2}-\mathrm{s}^{2}\right) /(4 \mathrm{~s}-3 \psi-\mathrm{sc}) .
\end{aligned}
$$

For $m=4, u_{1}, u_{2}$ are zeros of the quadratic $h_{2} u^{2}+h_{1} u+h_{0}$, where

$$
\begin{aligned}
& h_{2}=\mathrm{s}^{2}-\psi(\mathrm{sc}+\psi) / 2, \\
& h_{1}=(\mathrm{s} / 2)\left(\psi-\mathrm{sc}-2 \mathrm{~s}^{2} \psi / 3\right), \quad h_{0}=\left(\psi^{2}+2 \mathrm{sc} \psi-\mathrm{s}^{2}\left(3-\mathrm{s}^{2} / 3\right)\right) / 4, \\
& \beta_{1}=\left(\psi u_{2}-\mathrm{s}\right) /\left(u_{2}-u_{1}\right), \quad \beta_{2}=\left(\mathrm{s}-\psi u_{1}\right) /\left(u_{2}-u_{1}\right) .
\end{aligned}
$$

A few experiments have been run to compare the three- and four-point members of this family with the corresponding Legendre formulas. On the problem $\int_{-\pi / 2}^{\pi / 2}(\cos 1.5 x+\cos 2.5 x) d x$, the absolute error for the three-point Legendre quadrature exceeded that for the trigonometric method by a factor of 3.4 ; in the four-point case the factor was 7.8 . On the problem $\int_{-\pi / 2}^{\pi / 2} x^{2} \cos 2 x$, results favored the trigonometric method by factors of 1.4 and 3.3. Another experiment consisted in approximating $\ln x$ by applying quadrature formulas to

$$
\int_{-(x-1) / 2}^{(x-1) / 2}(1 /(z+(x+1) / 2)) d z
$$

at nine equal-spaced $x$-values in [.55, .95]. These results uniformly favored Legendre, but in no case did the absolute error for the trigonometric formula exceed that for Legendre by as much as a factor of 1.3. Both three-point methods yielded at least four correct decimal digits; the four-point methods always gave at least six correct digits. In short, the trigonometric methods seem to be quite closely competitive with Legendre in problems which are not deliberately biased towards one method or the other. It may be anticipated that the trigonometric methods will find their principal area of application in situations when the integrand is known to be (nearly) 
periodic; the period can then be normalized to $2 \pi$, so that the formulas become immediately applicable.

Boeing Company

Renton, Washington 98055

1. E. Isaacson \& H. B. Keller, Analysis of Numerical Methods, Wiley, New York, 1966. MR 34 \#924.

2. G. Birkhoff, D. M. Young \& E. H. Zarantonello, Numerical Methods in Conformal Mapping, Proc. Sympos. Appl. Math., Vol. 4; Fluid Dynamics, McGraw-Hill, New York, 1953, pp. 117-140. MR 15, 258.

3. F. B. Hildebrand, Introduction to Numerical Analysis, McGraw-Hill, NewYork, 1956. MR 17, 788 . 